\title{
APPLICATION OF A PROBABILITY MODEL BASED ON PARIS' LAW IN ASSESSING FATIGUE LIFE OF MARINE HIGH-STRENGTH STEEL STRUCTURES
}

Paris' law is a commonly used formula in the assessment of fatigue life of structures. In the present study, we sought to improve the accuracy of this law in marine engineering using a probability model to experimentally assess fatigue life. First, a fatigue crack growth test was performed using compact tension specimens made of marine high-strength steel to obtain a reasonable range of material coefficients $C$ and $m$ in Paris' law. The material coefficient $m$ was then determined as a constant, $C$ followed a log-normal distribution, and Paris' law with different probabilities dependent on $C$ was established. Finally, the probability model based on Paris' law was used to calculate fatigue life of a welded component with a surface crack caused by combined compressive and bending stresses. The results were then compared with the data from a fatigue test that used a crack front marking technique to depict the fractography. The findings of this study show a linear relationship between $\log C$ and $m$ that caused a mutual constraint of the material coefficients. In summary, the structure fatigue life calculated by the probability mode based on Paris' law is reliable, thus it can greatly improve safety and accuracy of fatigue life assessment in marine engineering.

Key words: $\quad$ fatigue life assessment, Paris' law, probability model, surface crack, experiment

\section{Introduction}

Fatigue damage is a major structural failure in ships and marine engineering. This problem is more serious as high-strength steel is widely used in deep sea equipment and warships [1][2]. Currently, researchers have adopted a method based on fatigue crack growth to accurately assess fatigue life of structures [3][4].

Several studies have explored various expressions for simulating stable fatigue crack propagation, however Paris' law is the most commonly known and used expression [5][6]:

$$
\frac{\mathrm{d} a}{\mathrm{~d} N}=C(\Delta K)^{m}
$$

where $C$ and $m$ are material coefficients, $a$ is the crack length, $N$ is the number of load cycles, $\mathrm{d} a / \mathrm{d} N$ is the fatigue crack growth rate, and $K$ is the stress intensity factor (SIF). $\Delta K$ is the SIF range and is calculated as shown below.

$$
\Delta K=K_{\max }-K_{\min }
$$


where $K_{\max }$ and $K_{\min }$ are the maximum and the minimum SIF in one load cycle, respectively.

Paris' law states that the fatigue crack growth rate is correlated with the range of SIF. By integrating Eq. (1), fatigue life can be expressed as the total number of load cycles during crack propagation from initial to critical crack lengths.

$$
N=\int_{a_{0}}^{a_{c}} \frac{\mathrm{d} a}{C(\Delta K)^{m}}
$$

where $a_{0}$ and $a_{c}$ denote the initial and critical crack lengths, respectively.

Four key factors, namely the material coefficients, the SIF, the initial and the critical crack lengths, are considered when assessing fatigue life using Paris' law. The initial crack length is generally determined using non-destructive testing (NDT) or related specifications, whereas the critical crack length is usually determined by fracture toughness $\left(K_{I C}\right)$ or related specifications. Some SIF expressions are reported in the SIF handbook. In particular cases, the SIF can be obtained using the finite element method, boundary element or weight function methods, among others [7-10]. Coefficients $C$ and $m$ in common materials are reported in the material handbook, whereas those of new materials are generally obtained from a fatigue crack growth rate test.

In practice, both $C$ and $m$ in Paris' law are generally determined as constants (such as the mean value), which is not safe. Actually, the material coefficients obtained by conducting the test usually have a dispersion. This dispersion is attributed to the internal dispersion of the material, such as internal microstructure and mechanical properties, or it can be caused by external errors of experimental conditions, such as specimen size, measurement technology and test environment. Previous studies report on several experiments and statistical analyses on material coefficients of Paris' law [11-17]. An accepted conclusion is that coefficient $m$ in Paris' law is normally distributed, whereas the other coefficient $C$ follows a log-normal distribution. Therefore, fatigue life at different safety probability can be obtained by using Paris' law with either a single random or double random variable.

Generally, components commonly used in marine engineering are subjected to combined compressive and bending stresses. For practical reasons, based on the experiment, this study simplifies the theory and focuses on the probability model of fatigue life assessment for welded components made of high-strength steel.

\section{Fatigue crack growth rate test}

Material coefficients in Paris' law can be obtained using a fatigue crack growth rate test. This test is performed with an electric servo-hydraulic material test system (MTS-New810), using compact tension specimens made of marine high-strength steel (Fig. 1). The material mechanical properties are as follows: ultimate strength is over $950 \mathrm{MPa}$, yield strength is over $900 \mathrm{MPa}$, Young's modulus is $200 \mathrm{GPa}$, and Poisson's ratio is 0.3 . The chemical composition of the material is summarized in Table 1.

Table 1 Chemical composition of Ni-Mn-Cr-Mo steel

\begin{tabular}{ccccccccc}
\hline Element & $\mathrm{Fe}$ & $\mathrm{C}$ & $\mathrm{Si}$ & $\mathrm{Mn}$ & $\mathrm{Ni}$ & $\mathrm{Cr}$ & $\mathrm{Mo}$ & $\mathrm{V}$ \\
Weight $(\%)$ & 93 & 0.08 & 0.30 & 0.65 & 4.80 & 0.61 & 0.54 & 0.068 \\
\hline
\end{tabular}




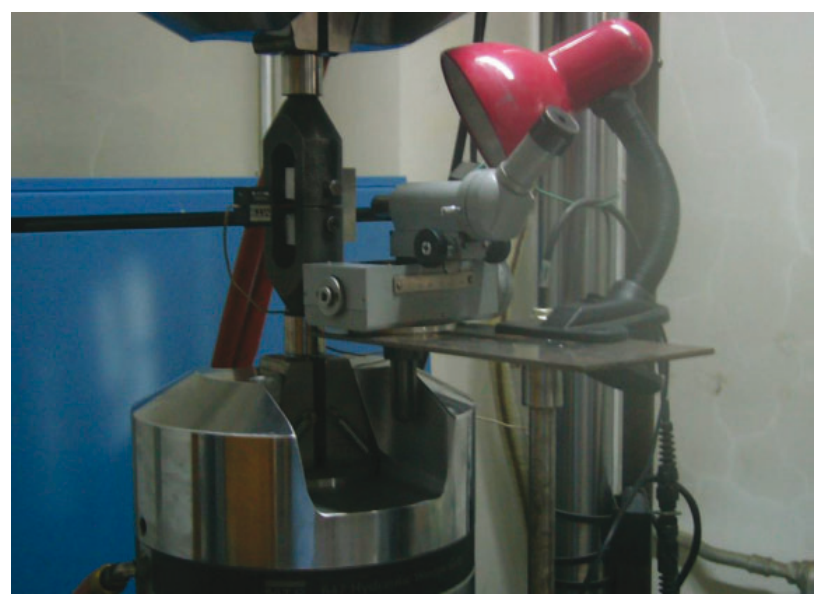

(a) Test set-up

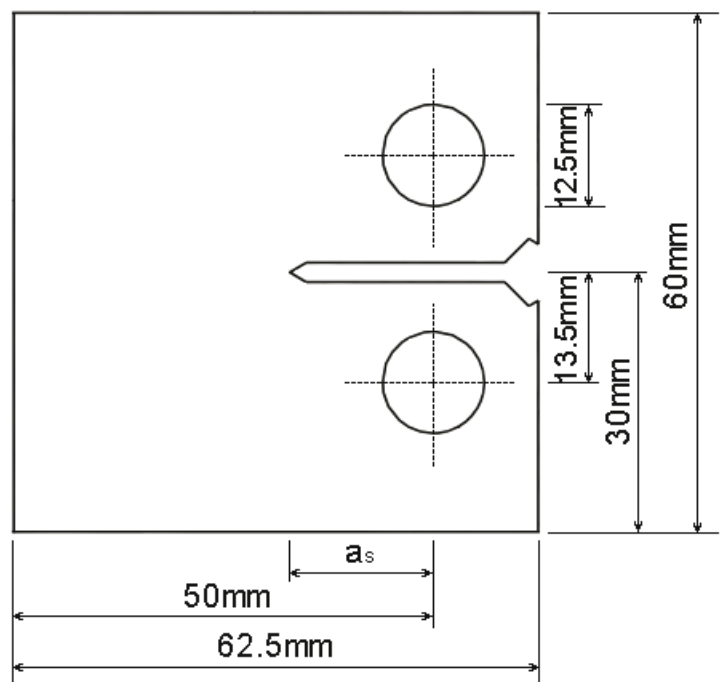

(b) Compact tension specimen

Fig. 1 Fatigue crack growth rate test

Table 2 Test conditions

\begin{tabular}{|c|c|c|c|c|c|}
\hline No. & $\begin{array}{c}B \\
/ \mathrm{mm}\end{array}$ & $\begin{array}{c}a_{\mathrm{s}} \\
/ \mathrm{mm}\end{array}$ & $\mathrm{R}$ & $\begin{array}{c}P \\
/ \mathrm{kN}\end{array}$ & Total number \\
\hline 1 & 25 & 20 & 0.1 & 15 & 6 \\
\hline 2 & 12 & 20 & 0.1 & 8 & 3 \\
\hline 3 & 12 & 20 & 0.1 & 7 & 3 \\
\hline 4 & 12 & 20 & 0.1 & 6 & 3 \\
\hline 5 & 12 & 12 & 0.3 & 10 & 3 \\
\hline 6 & 12 & 12 & 0.5 & 12 & 3 \\
\hline
\end{tabular}

where $B$ indicates the specimen thickness, $\mathrm{R}$ is the stress ratio, and $P$ is the applied load.

The sizes of the specimens are shown in Fig. 1(b) and Table 2. The frequency of the sinusoidal load was $10 \mathrm{~Hz}$. The total number of specimens is 21 , and the test conditions are shown in Table 2. The crack was prefabricated in a length of 2 to $4 \mathrm{~mm}$, and the crack length was measured using an optical microscope. The SIF range was calculated using the following equation:

$$
\Delta K=\frac{\Delta P}{B \sqrt{W}} \cdot \frac{(2+\alpha)}{(1-\alpha)^{1.5}}\left(0.886+4.64 \alpha-13.32 \alpha^{2}+14.72 \alpha^{3}-5.6 \alpha^{4}\right)
$$

where $\Delta P=P(1-\mathrm{R}), W$ is the specimen width, $\alpha=a / W$, and $a$ is the crack length.

The fatigue crack growth rate $\mathrm{d} a / \mathrm{d} N$ and the SIF range $\Delta K$ are obtained by fitting the test data using the seven-point incremental polynomial method. In the stage of stable crack propagation, studies report that the test data follows a linear relationship between $\log (\mathrm{d} a / \mathrm{d} N)$ and $\log (\Delta K)[18]$.

Eq. (5) is obtained by taking the logarithm of both sides of Paris' law as follows:

$$
\log (\mathrm{d} a / \mathrm{d} N)=\log C+m \log (\Delta K)
$$

According to Eq. (5), the material parameters $m$ and $C$ of each specimen are obtained by fitting the $\mathrm{d} a / \mathrm{d} N-\Delta K$ curves. 
The statistical distribution of the material coefficients in Paris' law is studied in the following two steps:

(1) Determining the correlation between $C$ and $m$, both of which are random variables. Assuming that $m$ conforms to normal distribution, $C$ is calculated through the correlation analysis. This step reveals a reasonable distribution of the material coefficients.

(2) Coefficient $m$ is determined as a constant, by selecting from the distribution in step 1. Coefficient $C$ is a random variable and follows a log-normal distribution. The probability model of Paris' law with single random variable can be obtained by this step.

\section{Reasonable distribution of material coefficients}

To obtain a correlation between $C$ and $m$ Eq. (1) is transformed into the following formula [19]:

$$
\frac{\mathrm{d} a}{\mathrm{~d} N}=C_{0}\left(\frac{\Delta K}{K_{0}}\right)^{m}
$$

where $K_{0}$ is a normalization factor and has the same dimension as $\Delta K$. The dimension of $C_{0}$ is a crack growth rate independent of the value of $m$, while $C_{0}$ and $K_{0}$ are both constants.

Then, the relationship between material coefficients $C$ and $m$ can be obtained using Eqs. (1) and (6):

$$
C=C_{0} K_{0}^{-m}
$$

Taking the logarithm of both sides of Eq. (7):

$$
\log C=\log C_{0}-m \log K_{0}
$$

From another point of view, if $C$ and $m$ are both variables, there is a linear relationship between $\log C$ and $m$.

The values of $m$ and $C$ of each specimen are obtained by fitting the $\mathrm{d} a / \mathrm{d} N-\Delta K$ curves. The linear relationship between $\log _{10} C$ and $m$ using the data is shown in Fig. 2.

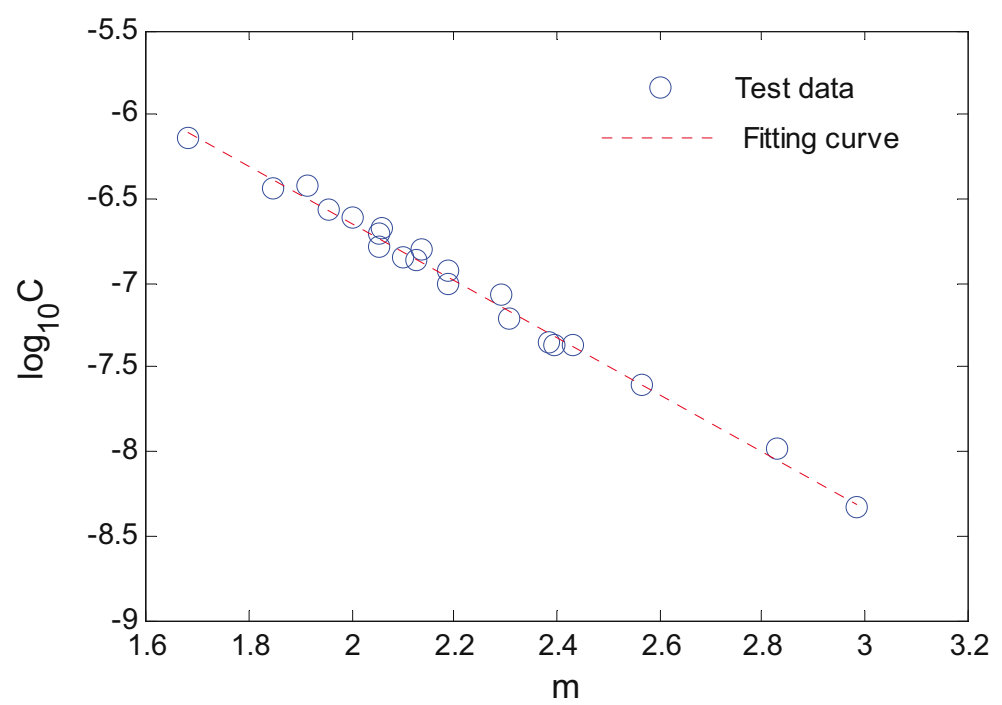

Fig. 2 Linear relationship between $\log _{10} C$ and $m$ 
The equation of the linear relationship between $\log _{10} C$ and $m$ is obtained using the least square method.

$$
\log _{10} C=-1.6927 m-3.2563
$$

where $R^{2}=0.9920$, which represents the fitting correlation coefficient. The closer $R^{2}$ is to 1 , the more accurate the fitting is.

The material coefficients $C$ and $m$ in Paris' law have a mutual constraint because of the linear relationship (Fig. 2 and Eq. (9)). One value is determined, whereas the other can be obtained from the correlation.

Previous studies report that coefficient $m$ follows a normal distribution. Therefore, the probability density of $m$ is obtained by using the test data.

$$
f(m)=1.2778 \exp \left[-\frac{(m-2.2136)^{2}}{0.1949}\right]
$$

The distribution of $m$ is normal as shown by the Kolmogorov-Smirnov test, $d_{\text {max }}=0.1469 \prec d_{(21,0.05)}=0.2872$.

According to the $3 \sigma$ principle of the normal distribution theory, the probability of coefficient $m$ in $(1.2770,3.1502)$ is $99.74 \%$. Meanwhile, the corresponding interval of coefficient $C$ is in $\left(2.5784 \times 10^{-9}, 3.8205 \times 10^{-6}\right)$. This represents a reasonable range of material coefficients in Paris' law. Values of material coefficients $C$ and $m$ cannot be arbitrarily determined due to the correlation between them.

\section{Probability model of Paris' law}

When coefficient $m$ is determined as a constant (selecting from Eq.10), $C$ can be obtained by using Eq. (11).

$$
\log _{10} C=\log _{10}(\mathrm{~d} a / \mathrm{d} N)-m \log _{10}(\Delta K)
$$

Fig. 2 shows that coefficient $m$ is mainly concentrated between 1.9 and 2.5. In this paper, the mean value of $m=2.2136$. Therefore, a total of 247 data values of $\log _{10} C$ are obtained from the test according to Eq. (11). The statistics of $\log _{10} C$ is shown in Table 3.

Table 3 Statistics of $\log _{10} C$

\begin{tabular}{ccc}
\hline Value & Occurrence number $(\mathrm{N})$ & Frequency of occurrence $(\mathrm{N} / 247)$ \\
\hline$>-6.913$ & 19 & 0.07692308 \\
$-6.913 \sim-6.973$ & 55 & 0.22267206 \\
$-6.973 \sim-7.033$ & 97 & 0.39271255 \\
$-7.033 \sim-7.093$ & 63 & 0.25506073 \\
$<-7.093$ & 13 & 0.05263158 \\
\hline
\end{tabular}

The statistical histogram of $\log _{10} C$ is shown in Fig. 3 . 


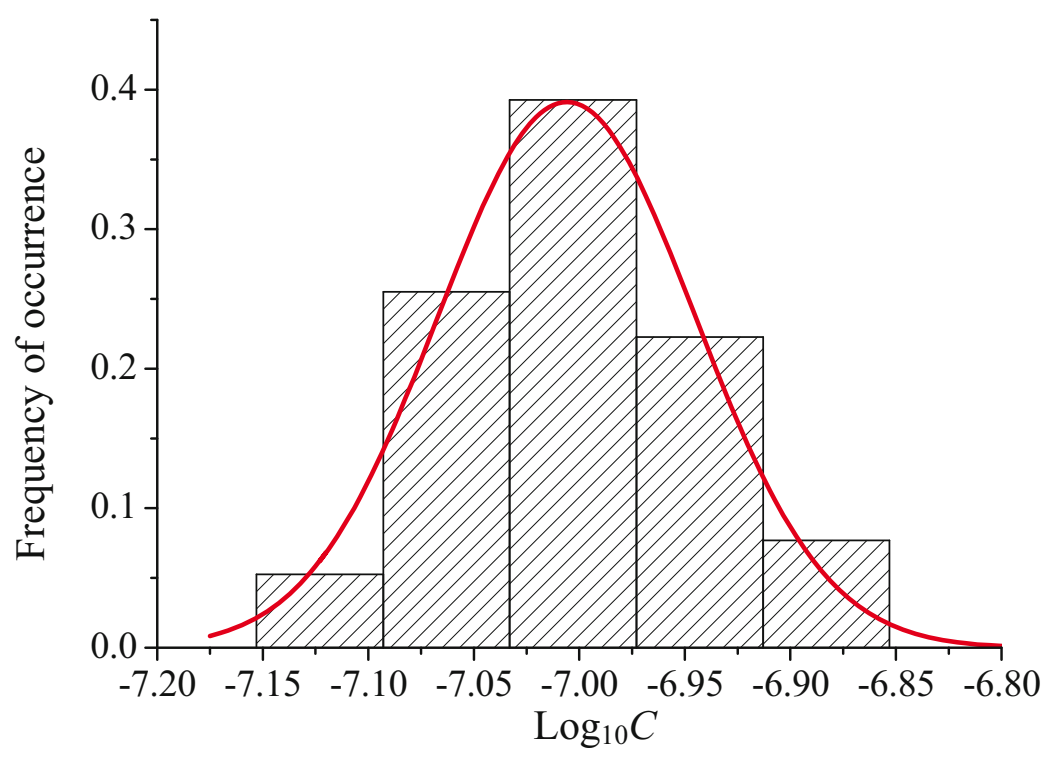

Fig. 3 Statistical histogram of $\log _{10} C$

The histogram shows that coefficient $C$ is in conformity to log-normal distribution when $m$ is determined as a constant.

Assuming that $x=\log _{10} C$, then the probability density of $\log _{10} C$ can be obtained.

$$
f(x)=6.5344 \exp \left[-\frac{(x+7.0028)^{2}}{0.0075}\right]
$$

When $m=2.2136, \log _{10} C$ at different safety probability is given as follows:

$$
x_{p}=\bar{x}+u_{p} \times \sigma
$$

where $\bar{x}=-7.0028, \sigma=0.0611 . p$ denotes a given safety probability, whereas $u_{P}$ is the standard normal deviation when the safety probability is $p$.

Therefore, the probability model of the fatigue life assessment based on Paris' law is obtained by:

$$
\frac{\mathrm{d} a}{\mathrm{~d} N}=10^{-7.0028+0.0611 * u_{p}}(\Delta K)^{2.2136}
$$

The physical interpretation of Eq. (14) is the following: the crack growth rate calculated by Paris' law is greater than or equal to the real crack growth rate, and the corresponding probability is $p$. For further interpretation, the fatigue life obtained by Eq. (14) is less than or equal to the real fatigue life, and the probability is $p$.

When $p=50 \%$, the material coefficients in Paris' law represent the mean value, and this is the mostly used model for assessing fatigue life in engineering. Other safety probabilities of the fatigue life assessment are generally $90 \%, 95 \%, 99 \%$, or $99.9 \%$. Some commonly used $u_{P}$ values are shown in Table 4 , whereas more values are found in the table of standard normal distribution. 
Table4 $p-u_{p}$

\begin{tabular}{ccccccccc}
\hline$p$ & $1 \%$ & $50 \%$ & $84.1 \%$ & $90 \%$ & $95 \%$ & $99 \%$ & $99.9 \%$ & $99.99 \%$ \\
\hline$u_{p}$ & -2.326 & 0 & 1 & 1.282 & 1.645 & 2.326 & 3.09 & 3.719 \\
$C\left(\times 10^{-8}\right)$ & 7.1650 & 9.9364 & 11.4362 & 11.8986 & 12.5216 & 13.7796 & 15.3420 & 16.7603 \\
$m$ & 2.2136 & 2.2136 & 2.2136 & 2.2136 & 2.2136 & 2.2136 & 2.2136 & 2.2136 \\
\hline
\end{tabular}

The $p-\mathrm{d} a / \mathrm{d} N-\Delta K$ curves are plotted in Fig. 4. These values show that the test data are basically distributed in the range of $p=1 \%$ to $p=99 \%$.

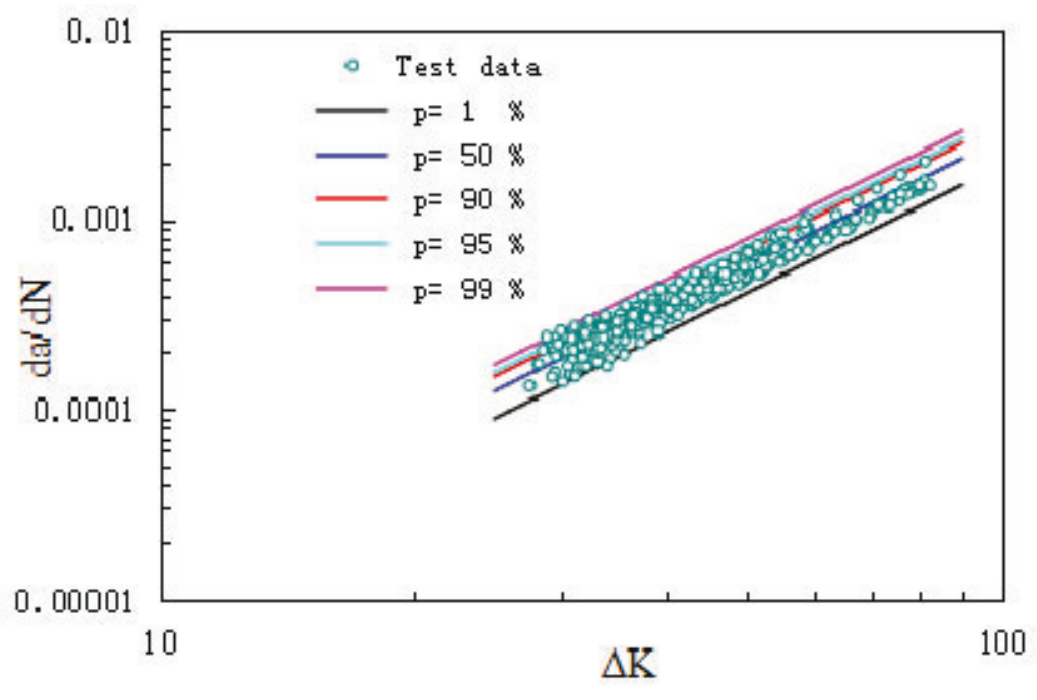

Fig. $4 p-\mathrm{d} a / \mathrm{d} N-\Delta K$

\section{Fatigue life assessment of welded components with a surface crack}

5.1 Welded components with a surface crack

Marine structures generally consist of various welded steel components, which are usually subjected to tensile, compressive or bending stresses in the ocean environment. Fatigue cracks usually initiate or propagate under alternating stresses. In addition to the cracks caused by material defects, most fatigue cracks may occur on the surface of components due to stress concentration or geometrical discontinuities. Therefore, the surface crack-related fracture failure is a common phenomenon in engineering.

To assess the fatigue life of welded components with surface cracks, we performed a fatigue test on samples using the MTS-New810 tool (Fig. 5). The sample comprised two butt-welded high-strength steel plates, and was subjected to combined compressive and bending stresses. Each plate measured $160 \times 80 \times 20 \mathrm{~mm}$, whereas the angle between the two plates was $160^{\circ}, 170^{\circ}$ and $180^{\circ}$, respectively. The samples met quality standards after defect detection, finishing and polishing. The pre-existing crack perpendicular to the axis of the sample was prefabricated by a mill at the weld toe. Thereafter, a sinusoidal load was applied to both ends of the sample, with load frequency and stress ratio set at $1 \mathrm{~Hz}$ and 0.1 , respectively. 


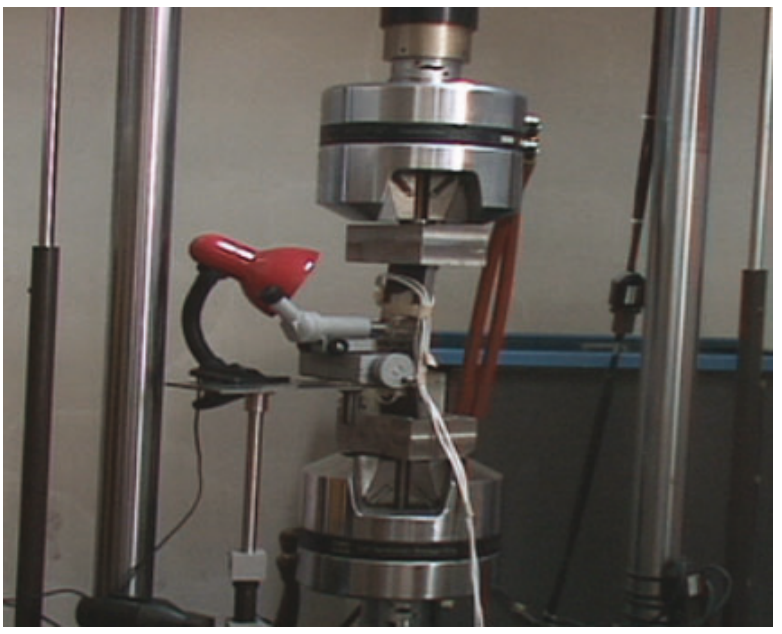

(a) Test set-up
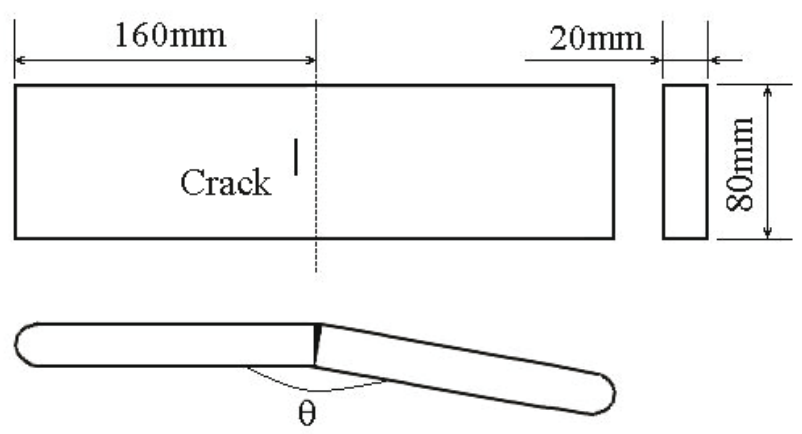

(b) Sample

Fig. 5 Fatigue test

There were three groups of samples. The crack front marking technique was used to depict the fractography, then CAD was applied to describe the fractography with beach marks and for the determination of the dimensions, as shown in Fig. 6.

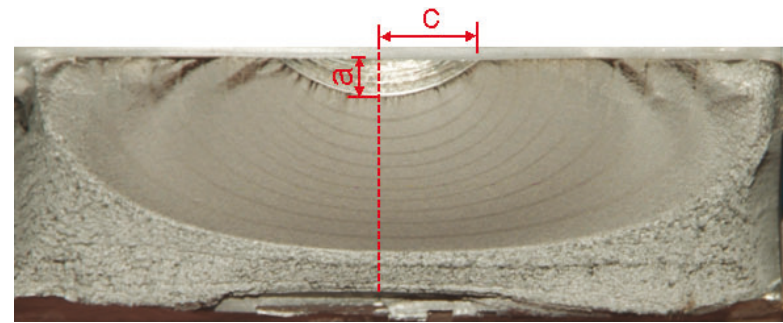

(a) Beach marks

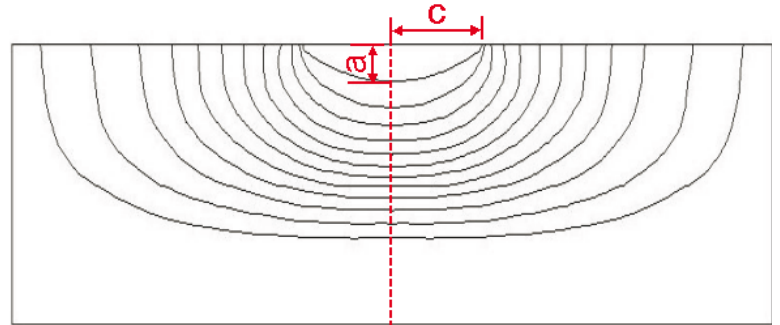

(b) Drawing by CAD

Fig. 6 Fractography of the sample (T3-1)

\subsection{Stress intensity factor}

The surface crack propagation is a series of circular arcs with continuously changing radius of curvature (Fig. 6). Newman and Raju's theory was used to quantitatively describe the change in the surface crack propagation under the combined compressive and bending stresses [20]. The analysis showed a relationship between the depth and the length of the semi-elliptic surface crack during the propagation. This relationship is expressed using Paris' law as follows:

$$
\left\{\begin{array}{l}
\frac{\mathrm{d} a}{\mathrm{~d} N}=C\left(\Delta K_{a}\right)^{m} \\
\frac{\mathrm{d} c}{\mathrm{~d} N}=C\left(0.9 \Delta K_{c}\right)^{m}
\end{array}\right.
$$

where $a$ and $c$ denote the depth and the half-length of the surface crack, respectively.

The SIF is calculated using the following equation:

$$
K=\left(\sigma_{0}+H \sigma_{b}\right) \frac{\sqrt{\pi a}}{\Phi} F\left(\frac{a}{c}, \frac{a}{t}, \frac{2 c}{W}, \varphi\right)
$$


where $\sigma_{0}$ indicates the tensile stress or the compressive stress, $\sigma_{b}$ is the bending stress, $t$ and $W$ denote the thickness and the width of the plate, respectively. $\varphi$ is the parametric angle of the semi-elliptical surface crack, which is used to define the crack front. When $\varphi=90^{\circ}$, it implies that the crack is propagating in the depth direction, whereas when $\varphi=0^{\circ}$, it means that the crack is propagating in the length direction. Other explanations are as follows:

$$
\begin{aligned}
& \Phi=\left[1.0+1.464(a / c)^{1.65}\right]^{1 / 2} \\
& F\left(\frac{a}{c}, \frac{a}{t}, \frac{2 c}{\omega}, \varphi\right)=\left[M_{1}+M_{2}(a / t)^{2}+M_{3}(a / t)^{4}\right] f_{\varphi} g f_{W} \\
& M_{1}=1.13-0.09(a / c) \\
& M_{2}=-0.54+0.89 /(0.2+a / c) \\
& M_{3}=0.5-1 /(0.65+a / c)+14(1-a / c)^{24} \\
& f_{\varphi}=\left[\sin ^{2} \varphi+(a / c)^{2} \cos ^{2} \varphi\right]^{1 / 4} \\
& g=1+\left[0.1+0.35(a / t)^{2}\right](1-\sin \varphi)^{2} \\
& f_{W}=\left[\sec \left(\frac{\pi}{2} \frac{2 c}{W} \sqrt{\frac{a}{t}}\right)\right]^{1 / 2} \\
& H=H_{1}+\left(H_{2}-H_{1}\right) \sin ^{p} \varphi \\
& H_{1}=1-0.34(a / t)-0.11(a / c)(a / t) \\
& H_{2}=1+G_{1}(a / t)+G_{2}(a / t)^{2} \\
& G_{1}=-1.22-0.12(a / c) \\
& G_{2}=0.55-1.05(a / c)^{0.75}+0.47(a / c)^{1.5} \\
& p=0.2+a / c+0.6(a / t)
\end{aligned}
$$

where $0<a / c \leq 1.0,0 \leq a / t \leq 1.0,2 c / W \leq 1$, and $0 \leq \varphi \leq \pi$.

The applicability of Newman \& Raju's SIF is verified by comparing the test data and the calculated results, as shown in Table 5. To eliminate the influence of crack initiation, the first beach marks are defined as the initial crack in the calculation, and the material coefficients are set as follows: $m=2.2136$ and $C=9.9364 \times 10^{8}$ (the mean value from Table 4). This implies that Newman \& Raju's theory can highly simulate surface crack propagation under combined compressive and bending stresses. 
Table 5 Comparison between test data and calculated results ( T3-1 )

\begin{tabular}{ccccc}
\hline & & Test data & & \multicolumn{2}{c}{ Calculated value } \\
Beach marks & $a / \mathrm{mm}$ & $c / \mathrm{mm}$ & $c / \mathrm{mm}$ & Error \\
\hline Pre-existing crack & 2.56 & 9.70 & - & - \\
Beach mark-1 & 4.36 & 10.05 & 11.10 & $5.21 \%$ \\
Beach mark-2 & 5.64 & 10.55 & 12.41 & $7.35 \%$ \\
Beach mark-3 & 6.77 & 11.56 & 13.83 & $8.22 \%$ \\
Beach mark-4 & 7.71 & 12.78 & 15.43 & $5.76 \%$ \\
Beach mark-5 & 8.55 & 14.59 & 17.2 & $3.49 \%$ \\
Beach mark-6 & 9.32 & 16.62 & 19.36 & $2.38 \%$ \\
Beach mark-7 & 10.1 & 18.91 & 22.09 & $2.17 \%$ \\
Beach mark-8 & 10.91 & 21.62 & 25.65 & $3.55 \%$ \\
Beach mark-9 & 11.77 & 24.77 & 31.03 & $4.58 \%$ \\
Beach mark-10 & 12.8 & 29.67 & 37.43 & $3.37 \%$ \\
Fracture failure & 13.76 & 36.21 & - & - \\
\hline
\end{tabular}

\subsection{Fatigue life under different probabilities}

Fatigue life of the surface cracks is calculated using the iteration method. In summary, the crack propagation from initial crack depth $a_{0}$ to critical crack depth $a_{c}$ is divided into several small stages $\Delta a=a_{i+1}-a_{i}$, then the fatigue life of crack propagation from $a_{i}$ to $a_{i+1}$ is calculated using the following equation:

$$
N_{i}=\int_{a_{i}}^{a_{i+1}} \frac{\mathrm{d} a}{C(\Delta K)^{m}}=\frac{\Delta a}{C(\Delta K)^{m}}
$$

The fatigue life of crack propagation from $a_{0}$ to $a_{c}$ is obtained by iteration.

$$
N=\sum N_{i}
$$

The value of the initial defect is determined by conducting a test. In summary, $a_{0}=0.25 \mathrm{~mm}$ and $c_{0}=1.0 \mathrm{~mm}$. The sample is regarded as failure when $2 c / W \succ 0.5$, while the corresponding crack depth is the critical crack depth $a_{c}$. The material coefficients in Paris' law are derived from Table 4. The fatigue life of the samples under different safety probabilities is shown in Table 6 .

Table 6 Fatigue life of the samples

\begin{tabular}{cccccccccc}
\hline Sample Angle & $\begin{array}{c}\text { Bending } \\
\text { stress } \\
\text { /MPa }\end{array}$ & $\begin{array}{c}\text { Compressive } \\
\text { stress } \\
\text { /MPa }\end{array}$ & $\begin{array}{c}\text { Fatigue life } \\
\text { resulting from } \\
\text { test }\end{array}$ & \multicolumn{2}{c}{ Fatigue life resulting from theoretical calculation } \\
$p=1 \%$ & $p=50 \%$ & $p=90 \%$ & $p=95 \%$ & $p=99 \%$ \\
\hline $\mathrm{T} 1-1$ & $160^{\circ}$ & 647 & 45 & 31,855 & 38,787 & 27,969 & 23,357 & 22,195 & 20,168 \\
$\mathrm{~T} 1-2$ & $160^{\circ}$ & 647 & 45 & 17,928 & 31,430 & 22,663 & 18,926 & 17,985 & 16,343 \\
$\mathrm{~T} 2-1$ & $170^{\circ}$ & 684 & 35 & 25,945 & 29,669 & 21,394 & 17,866 & 16,977 & 15,427 \\
$\mathrm{~T} 2-2$ & $170^{\circ}$ & 604 & 25 & 32,988 & 39,613 & 28,565 & 23,854 & 22,667 & 20,598 \\
$\mathrm{~T} 3-1$ & $180^{\circ}$ & 525 & 0 & 33,155 & 40,678 & 29,333 & 24,495 & 23,276 & 21,151 \\
$\mathrm{~T} 3-2$ & $180^{\circ}$ & 557 & 0 & 28,609 & 39,360 & 28,382 & 23,702 & 22,522 & 20,466 \\
\hline
\end{tabular}

Note: fatigue life is expressed as a total number of load cycles. 
The test data show that the fatigue life of the samples under the same conditions has a specific dispersion. Overall, the fatigue life calculated using Paris' law with the mean value of material coefficients is an approximate of the test data. The fatigue life is therefore obtained using the mean value formula, while the corresponding safety probability of the component is $50 \%$. From a practical engineering point of view, the fatigue life of half of the components will be overestimated, which is not safe. However, as normal practice, a safety factor is added to the mean value formula, the reliability is unknown and the fatigue life tends to be conservative or dangerous. Therefore, fatigue life can be calculated by Paris' law at different safety probability based on an actual engineering requirement, thereby improving the safety and accuracy of the fatigue life assessment.

\section{Conclusions}

Prediction and assessment of fatigue life of structures is a major concern in engineering. Paris' law, which represents a simple approach to describing crack growth in the stable phase, is a commonly used formula for assessing fatigue life. In this study, we sought to improve the accuracy of this law and make the law applicable in the area of engineering by applying a probability model. From our experimental work, the following conclusions can be drawn:

(1) Material coefficients $C$ and $m$ in Paris' law obtained by test are scattered. Consequently, there is a linear relationship between $\log C$ and $m$, which causes a mutual constraint of these material coefficients. Therefore, when one is determined, the other can be obtained from the correlation.

(2) When $m$ is determined as a constant, $C$ follows a log-normal distribution. Therefore, the probability model of the fatigue life assessment based on Paris' law is dependent on material coefficient $C$ as a single random variable.

(3) The stress intensity factor based on Newman \& Raju's theory is highly accurate in simulating the surface crack propagation under combined compressive and bending stresses.

(4) Fatigue life can be calculated by Paris' law at different safety probability based on actual engineering requirements, thereby greatly improving safety and accuracy of fatigue life assessment.

\section{REFERENCES}

[1] Dong Q, Yang P, Xu G. Low cycle fatigue and ratcheting failure behavior of AH32 steel under uniaxial cyclic loading. International Journal of Naval Architecture and Ocean Engineering, 2019, 11(2), 671678. https://doi.org/10.1016/j.ijnaoe.2018.09.003

[2] Thompson I. Fatigue damage variation within a class of naval ships. Ocean Engineering, 2018, 165, 123130. https://doi.org/10.1016/j.oceaneng.2018.07.036

[3] Cui W C. A feasible study of fatigue life prediction for marine structures based on crack propagation analysis. Proceedings of the Institution of Mechanical Engineers, Part M: Journal of Engineering for the Maritime Environment, 2003, 217(1), 11-23. https://doi.org/10.1243/147509003321623112

[4] Yan X S, Huang X P, Cui W C. An engineering method to predict fatigue crack propagation life for marine structures. Journal of Ship Mechanics, 2016, 20(3), 323-334.

[5] Paris P C, Gomez M P, Anderson W E. A rational analytic theory of fatigue. The trend in engineering, 1961, 13(1), 9-14.

[6] Paris P C, Erdogan F. A critical analysis of crack propagation laws. Journal of Basic Engineering, 1963, 85D(1), 528-534. https://doi.org/10.1115/1.3656902

[7] Liao M M, Zhang P. An improved approach for computation of stress intensity factors using the finite element method. Theoretical and Applied Fracture Mechanics, 2019, 101, 185-190. https://doi.org/10.1016/j.tafmec.2019.02.019 
[8] Alatawi I A, Trevelyan J. A direct evaluation of stress intensity factors using the extended dual boundary element method. Engineering Analysis with Boundary Elements, 2015, 52, 56-63. https://doi.org/10.1016/j.enganabound.2014.11.022

[9] Yang S T, Ni Y L, Li C Q. Weight function method to determine stress intensity factor for semi-elliptical crack with high aspect ratio in cylindrical vessels. Engineering Fracture Mechanics, 2013, 109, 138-149. https://doi.org/10.1016/j.engfracmech.2013.05.014

[10] Li H, Guo R, Cheng H M. Calculation of stress intensity factors of matrix crack tip in particle reinforced composites using the singular Voronoi cell finite element method. Theoretical and Applied Fracture Mechanics, 2019, 101, 269-278. https://doi.org/10.1016/j.tafmec.2019.03.008

[11] Shen W, Soboyejo A B O, and Soboyejo W O. Probabilistic modeling of fatigue crack growth in Ti-6Al4V. International journal of fatigue, 2001, 23(10), 917-925. https://doi.org/10.1016/s0142-1123(01)00045-7

[12] Luo J, Bowen P. Statistical aspects of fatigue behaviour in a PM Ni-base superalloy Udimet. Acta materialia, 2003, 51(12), 3521-3535. https://doi.org/10.1016/s1359-6454(03)00171-x

[13] Wu W F, Ni C C. Statistical aspects of some fatigue crack growth data. Engineering Fracture Mechanics, 2007, 74(18), 2952-2963. https://doi.org/10.1016/j.engfracmech.2006.08.019

[14] Li W, Sakai T, Li Q, Wang P. Statistical analysis of fatigue crack growth behavior for grade B cast steel," Materials and Design, 2011, 32(3), 1262-1272. https://doi.org/10.1016/j.matdes.2010.09.036

[15] Carrascal I, Casado J A, Diego S, Lacalle R, Cicero S, Alvarez J A. Determination of the Paris" law constants by means of infrared thermographic technique. Polymer Testing, 2014, 40, 39-45. https://doi.org/10.1016/j.polymertesting.2014.08.005

[16] Fan X L, Zhao M D, Wang T J. Experimental investigation of the fatigue crack propagation in a closedcell aluminum alloy foam. Materials Science and Engineering A, 2017, 708, 424-431. https://doi.org/10.1016/j.msea.2017.09.120

[17] Mohammadipour A, Willam K. A numerical lattice method to characterize a contact fatigue crack growth and its Paris' coefficients using configurational forces and stress-life curves. Computer Methods in Applied Mechanics and Engineering, 2018, 340, 236-252.

[18] Božić Ž, Wolf H, Semenski D. Fatigue Growth of Multiple Cracks in Plates under Cyclic Tension. Transactions of FAMENA, 2010, 34(1), 1-12.

[19] Bergner F, and Zouhar G. A new approach to the correlation between the coefficient and the exponent in the power law equation of fatigue crack growth. International Journal of Fatigue, 2001, 22(3), 229-239. https://doi.org/10.1016/s0142-1123(99)00123-1

[20] Newman J C, and Raju I S. An empirical stress-intensity factor equation for the surface crack. Engineering Fracture Mechanics, 1981, 15(1-2), 185-192. https://doi.org/10.1016/0013-7944(81)90116-8

$\begin{array}{lll}\text { Submitted: } & 18.02 .2020 & \text { Jie Zhang* } \\ & & \text { Junfeng Gan } \\ \text { Accepted: } & 22.4 .2021 & \text { Ying Zeng } \\ & & \text { College of Ocean Science and } \\ & \text { Engineering, Shanghai Maritime } \\ & \text { University, Shanghai 201306, China. } \\ & \text { *Corresponding author: } \\ & \text { zhangjie@shmtu.edu.cn }\end{array}$

